\title{
Limb/pelvis-hypoplasia/aplasia syndrome (Al-Awadi/Raas-Rothschild syndrome): report of two Italian sibs and further confirmation of autosomal recessive inheritance
}

Gianni Camera, Giuseppe Ferraiolo, Domenico Leo, Alberto Spaziale, Silvano Pozzolo

\begin{abstract}
A third family with two Italian neonates affected with limb/pelvis-hypoplasia/aplasia syndrome is reported. The disorder shows autosomal recessive inheritance. (f Med Genet 1993;30:65-9)
\end{abstract}

Limb/pelvis-hypoplasia/aplasia syndrome was defined by Raas-Rothschild et al ${ }^{1}$ who reported three sibs with severe upper and lower limb malformations and a severely malformed pelvis. Previously, Al-Awadi et al had described two Arab sibs with profound limb deficiency, thoracic dystrophy, unusual facies, and normal intelligence. This report describes two new cases of limb/pelvis-hypoplasia/aplasia syndrome in Italian sibs.

\section{Case reports}

CASE 1

Case 1, a male, was the second child born to unrelated healthy parents at 40 weeks' gestation by caesarian section. Birth weight was $3700 \mathrm{~g}$ (50th to 70th centile), length $41 \mathrm{~cm}$ (<3rd centile), head circumference $34 \mathrm{~cm}$ (10th to 25 th centile), and chest circumference $36 \mathrm{~cm}$ (90th to 97th centile). His sister was healthy. There was no history of teratogenic exposure or drug ingestion during the pregnancy. The karyotype was normal $46, X Y$. Physical examination (fig 1) showed asymmetrical facies with epicanthic folds, large nose with naevus flammeus on the tip, large fishlike mouth, pointed chin, high and narrow palate, low set, dysplastic, and large ears; a short neck; a barrel shaped chest and pectus carinatum with increased distance between hypoplastic nipples; upwardly displaced external genitalia, glandular hypospadias, and hypoplastic scrotum with bilateral cryptorchidism; flexion contracture of the elbow joint and severe shortening and bowing of the forearms; very mobile wrist joints; four abnormally shaped digits on each hand with no fingernails; an upward and forward displaced, small pelvis, a pilonidal sinus, a deep dimple on the right thigh, and no gluteal cleft; and stick-like lower limbs with appendages resembling hypoplastic feet.

Radiographic examination (figs 2 and 3 ) showed a poorly ossified calvarium and pseudarthrosis of the right clavicle; traumatic fractured left humerus, shortened and bowed radii, absent ulnae, and hypoplasia/aplasia of the metcarpals and phalanges; underdeveloped iliac bones and absent pubic bones; aplastic femora, hypoplastic tibiae, absent fibulae, and hypoplasia/aplasia of the tarsals, metatarsals, and phalanges. Ultrasonographic examination showed no abnormalities of internal organs. The infant died aged 16 days of respiratory distress. No necropsy was performed.

CASE 2

Case 2, a female, was the sister of case 1 . Inexplicably, no fetal ultrasonography was performed before 30 weeks of gestation. At this time it showed a fetus with normal humeri, shortened and bowed radii, absent ulnae, and dysplastic, short lower limbs. The infant was delivered at 39 weeks by caesarian section. Birth weight was $3300 \mathrm{~g}$ (50th centile), birth length $41 \mathrm{~cm}$ ( $<3$ rd centile), head circumference $32.5 \mathrm{~cm}$ ( $3 \mathrm{rd}$ centile), and chest circumference $35 \mathrm{~cm}$ (90th centile). She showed a similar phenotype to case 1 (fig 4). Ultrasonographic examination indicated no abnormalities of the internal organs. $X$ ray examination (figs 5 and 6) showed a poorly ossified skull, pseudarthosis of the right clavicle, bowed radii, absence of the ulnae, hypoplastic pelvic bones, hypoplastic femora, slender tibiae, absent fibulae, and hypoplasia/ aplasia of the metacarpals, tarsals, metatarsals, and phalanges. The spine was considered to be normal.

The clinical and radiological findings in our patients, compared with those cases described by Al-Awadi $e t$ al ${ }^{2}$ and by Raas-Rothschild $e t$ $a l^{1}$ are reported in tables 1 and 2 .

\section{Discussion}

Congenital hypoplasia of the femur is the main sign in four uncommon disorders: limb/ pelvis-hypoplasia/aplasia syndrome, proximal femoral focal deficiency (PFFD), ${ }^{3}$ femurfibula-ulna (FFU) complex, ${ }^{4}$ and femoral hypoplasia-unusual facies syndrome. ${ }^{5}$ PFFD is a congenital absence of the proximal onethird to two-thirds of the femur and is often associated with ipsilateral absence of the fibula. FFU complex is characterised by absence of the proximal part of the femur with fibular defects and ulnar abnormalities. In the femoral hypoplasia-unusual facies syndrome, 


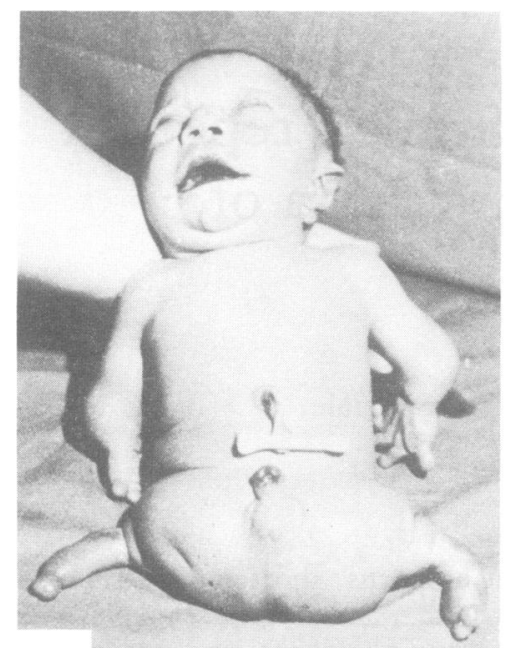

A
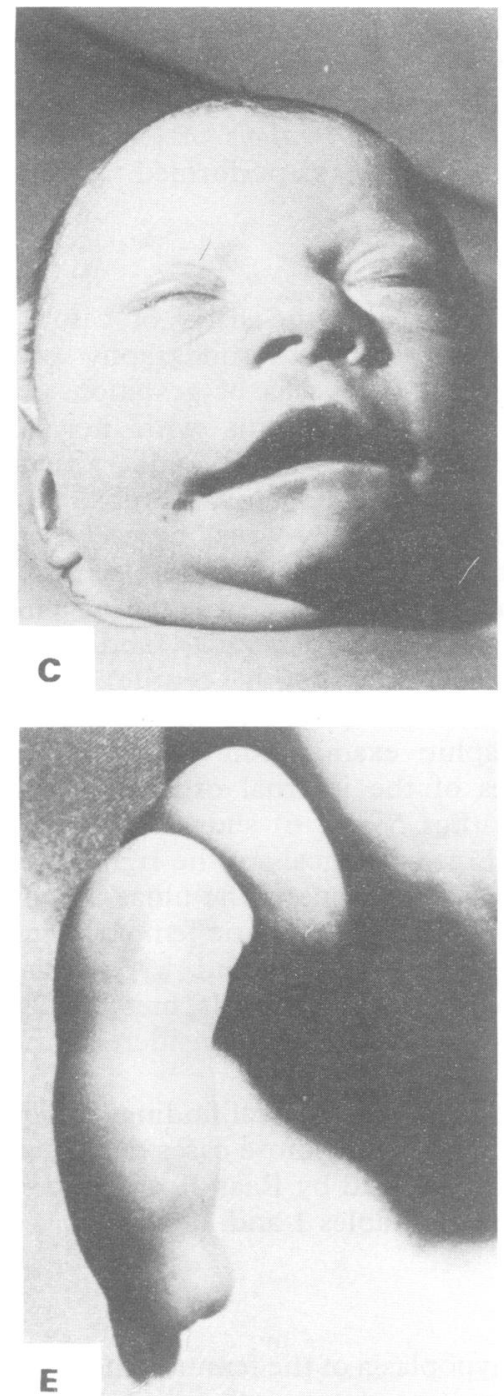
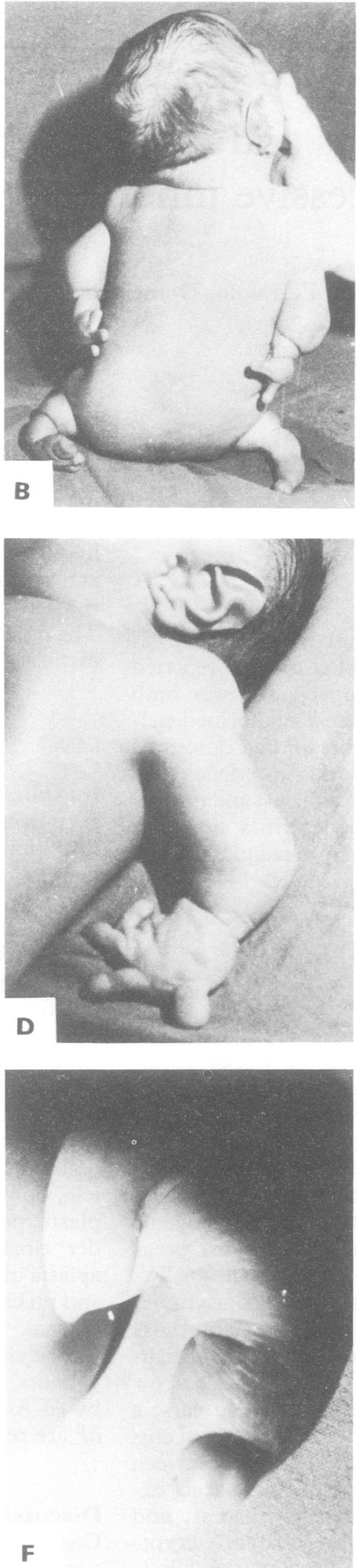

Figure 1 Case 1. $(A, B)$ patient's appearance, $(C)$ carp shaped mouth, pointed chin, (D) dysplastic large ear, severe malformations of upper limb, malformed digits, $(E, F)$ malformed lower limbs with appendages resembling poorly defined feet.

the femur ranges from being hypoplastic to aplastic and the facies show upward slanting palpebral fissures, short nose, long philtrum, and micrognathia.

The patients described here show upper and

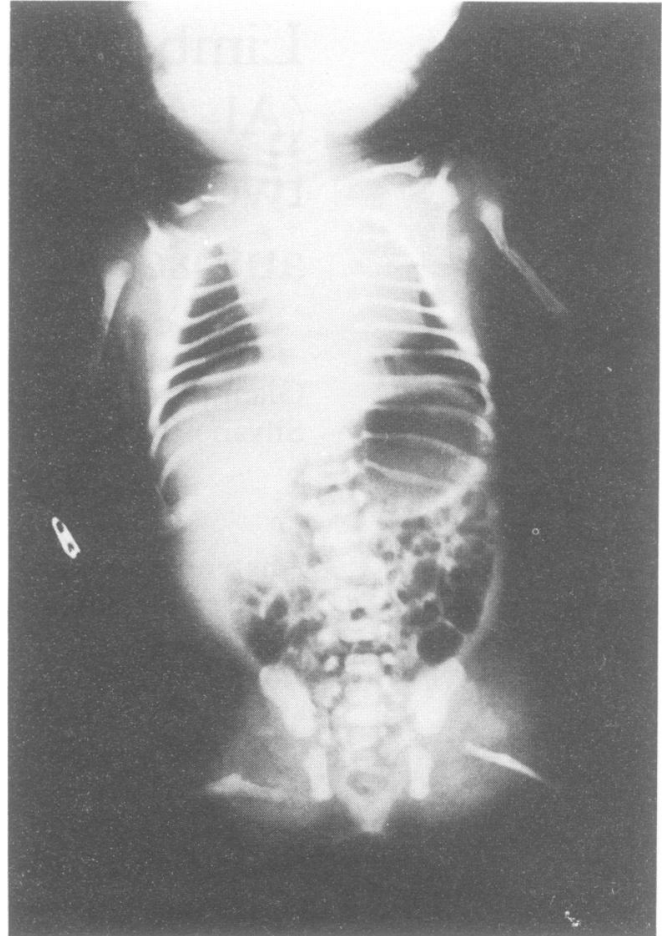

Figure 2 Case 1. Pseudarthrosis of the right clavicle, hypoplastic iliac bones, absent pubic bones, absent

femora, and thin tibiae.

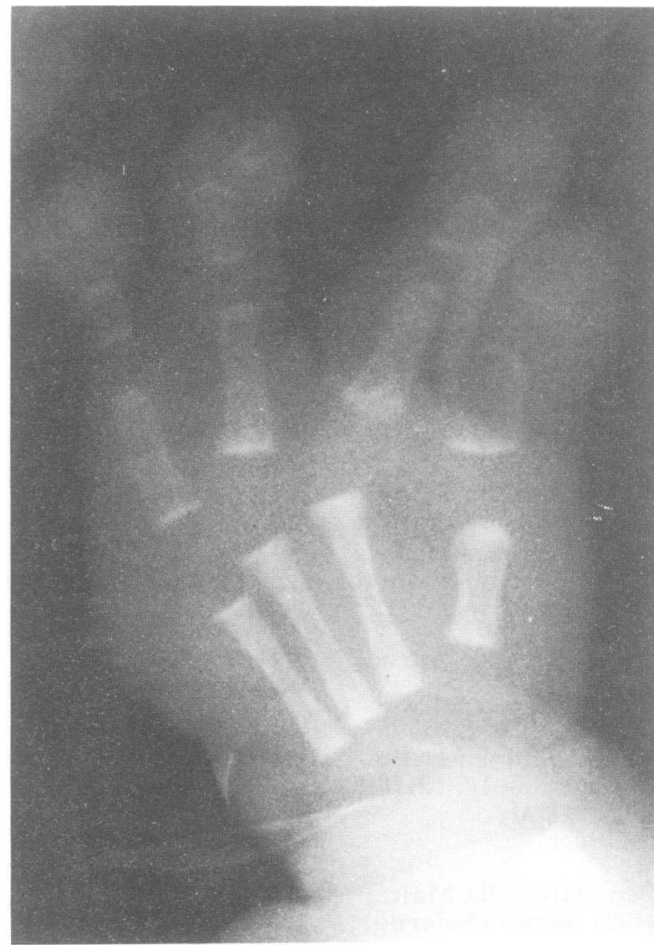

Figure 3 Case 1. Left hand with four malformed fingers.

lower limb malformations and malformed pelvis, resembling those reported in the patients with limb/pelvis-hypoplasia/aplasia syndrome. Our patients had features not described before, such as very dysplastic, large ears, high 
Table 1 Clinical findings in our patients compared with the cases reported by Al-Awadi et al ${ }^{2}$ and Raas-Rothschild et al.

\begin{tabular}{|c|c|c|c|c|c|c|c|}
\hline \multirow[b]{2}{*}{ Clinical findings } & \multicolumn{2}{|c|}{ Our cases } & \multicolumn{2}{|c|}{ Al-Awadi et al } & \multicolumn{3}{|c|}{ Raas-Rothschild et al } \\
\hline & $\begin{array}{c}\text { Case } \\
1\end{array}$ & $\begin{array}{c}\text { Case } \\
2\end{array}$ & $\begin{array}{c}\text { Case } \\
1\end{array}$ & $\begin{array}{c}\text { Case } \\
2\end{array}$ & $\begin{array}{c}\text { Case } \\
1\end{array}$ & $\begin{array}{c}\text { Fetus } \\
1\end{array}$ & $\begin{array}{c}\text { Fetus } \\
2\end{array}$ \\
\hline $\begin{array}{l}\text { Face } \\
\text { Asymmetrical } \\
\text { Elongated } \\
\text { Broad nasal bridge } \\
\text { Epicanthic folds } \\
\text { Dysplastic ears } \\
\text { Long ears } \\
\text { Pointed chin }\end{array}$ & $\begin{array}{l}+ \\
+ \\
+ \\
+ \\
+ \\
+ \\
+\end{array}$ & $\begin{array}{l}+ \\
+ \\
+ \\
+ \\
+ \\
+ \\
+\end{array}$ & $\begin{array}{l}- \\
+ \\
+ \\
+ \\
- \\
- \\
-\end{array}$ & $\begin{array}{l}- \\
+ \\
+ \\
- \\
- \\
-\end{array}$ & $\begin{array}{l}- \\
- \\
- \\
- \\
+ \\
+\end{array}$ & $\begin{array}{l}? \\
? \\
? \\
? \\
? \\
? \\
?\end{array}$ & $\begin{array}{l}? \\
? \\
? \\
? \\
? \\
? \\
?\end{array}$ \\
\hline $\begin{array}{l}\text { Thorax } \\
\text { Barrel shaped chest } \\
\text { Prominent sternum } \\
\text { Pectus carinatum } \\
\text { Hypoplastic nipples }\end{array}$ & $\begin{array}{l}+ \\
- \\
+ \\
+\end{array}$ & $\begin{array}{l}+ \\
- \\
+ \\
+\end{array}$ & $\begin{array}{l}+ \\
+ \\
- \\
-\end{array}$ & $\begin{array}{l}+ \\
+ \\
- \\
-\end{array}$ & $\begin{array}{l}- \\
- \\
-\end{array}$ & $\begin{array}{l}? \\
? \\
? \\
?\end{array}$ & $\begin{array}{l}? \\
? \\
? \\
?\end{array}$ \\
\hline $\begin{array}{l}\text { Upper limbs } \\
\text { Flexion contracture of elbow joint } \\
\text { Shortened forearm } \\
\text { Mobile wrist } \\
\text { Malformed digits }\end{array}$ & $\begin{array}{l}+ \\
+ \\
+ \\
+\end{array}$ & $\begin{array}{l}+ \\
+ \\
+ \\
+\end{array}$ & $\begin{array}{l}+ \\
+ \\
+ \\
+\end{array}$ & $\begin{array}{l}+ \\
+ \\
+\end{array}$ & $\begin{array}{l}+ \\
+ \\
+ \\
+\end{array}$ & $\begin{array}{l}+ \\
+ \\
? \\
+\end{array}$ & $\begin{array}{l}- \\
+ \\
? \\
+\end{array}$ \\
\hline $\begin{array}{l}\text { Pelvis } \\
\text { Severely malformed }\end{array}$ & + & + & + & + & + & + & + \\
\hline $\begin{array}{l}\text { External genitalia } \\
\text { Sex } \\
\text { Hypospadias } \\
\text { Hypoplastic scrotum } \\
\text { Inguinal testes } \\
\text { Upwardly displaced genitalia }\end{array}$ & $\begin{array}{l}M \\
+ \\
+ \\
+ \\
+\end{array}$ & $\begin{array}{l}\mathrm{F} \\
\mathrm{NR} \\
\mathrm{NR} \\
\mathrm{NR} \\
+\end{array}$ & $\begin{array}{l}\mathrm{F} \\
\mathrm{NR} \\
\mathrm{NR} \\
\mathrm{NR} \\
-\end{array}$ & $\begin{array}{l}M \\
- \\
- \\
+ \\
+\end{array}$ & $\begin{array}{l}M \\
- \\
+ \\
+ \\
+\end{array}$ & $\begin{array}{l}\text { F } \\
\text { NR } \\
\text { NR } \\
\text { NR } \\
+\end{array}$ & $\begin{array}{c}M \\
? \\
? \\
? \\
+\end{array}$ \\
\hline $\begin{array}{l}\text { Lower limbs } \\
\text { Stick-like projections } \\
\text { Hypoplastic feet }\end{array}$ & $\begin{array}{l}+ \\
+\end{array}$ & $\begin{array}{l}+ \\
+\end{array}$ & $\begin{array}{l}+ \\
+\end{array}$ & $\begin{array}{l}+ \\
+\end{array}$ & $\begin{array}{l}+ \\
+\end{array}$ & $\begin{array}{l}+ \\
+\end{array}$ & $\begin{array}{l}+ \\
+\end{array}$ \\
\hline $\begin{array}{l}\text { Other } \\
\text { Broad neck } \\
\text { Short neck } \\
\text { Hairy pigmented naevus } \\
\text { Pilonidal sinus } \\
\text { Deep anal pit } \\
\text { High and narrow palate } \\
\text { Occipital meningocele } \\
\text { Occipital neck tissue mass }\end{array}$ & $\begin{array}{l}+ \\
+ \\
+ \\
+ \\
+ \\
+ \\
-\end{array}$ & $\begin{array}{l}+ \\
+ \\
+ \\
+ \\
+ \\
+ \\
-\end{array}$ & $\begin{array}{l}+ \\
- \\
- \\
- \\
- \\
- \\
-\end{array}$ & $\begin{array}{l}+ \\
- \\
- \\
- \\
+ \\
- \\
-\end{array}$ & $\begin{array}{l}- \\
\overline{+} \\
+ \\
- \\
- \\
-\end{array}$ & $\begin{array}{l}- \\
- \\
- \\
- \\
- \\
- \\
+\end{array}$ & $\begin{array}{l}+ \\
+ \\
- \\
- \\
- \\
+ \\
-\end{array}$ \\
\hline
\end{tabular}

$+=$ present; $-=$ not present or not mentioned; ? = too early to know; NR= not relevant.

Table 2 Radiographical findings in our patients compared with the cases reported by $A l$-Awadi et al ${ }^{2}$ and Raas-Rothschild et al.'

\begin{tabular}{|c|c|c|c|c|c|c|c|}
\hline \multirow[b]{2}{*}{ Clinical findings } & \multicolumn{2}{|c|}{ Our cases } & \multicolumn{2}{|c|}{ Al-Awadi et al } & \multicolumn{3}{|c|}{ Raas-Rothschild et al } \\
\hline & $\begin{array}{c}\text { Case } \\
1\end{array}$ & $\begin{array}{c}\text { Case } \\
2\end{array}$ & $\begin{array}{c}\text { Case } \\
1\end{array}$ & $\begin{array}{c}\text { Case } \\
2\end{array}$ & $\begin{array}{c}\text { Case } \\
1\end{array}$ & $\begin{array}{c}\text { Fetus } \\
1\end{array}$ & $\begin{array}{c}\text { Fetus } \\
2\end{array}$ \\
\hline $\begin{array}{l}\text { Skull } \\
\text { Poorly ossified calvarium }\end{array}$ & + & + & - & - & - & - & - \\
\hline $\begin{array}{l}\text { Upper limbs } \\
\text { Shortened and bowed radii } \\
\text { Aplasia of ulna } \\
\text { Aplasia of carpal bones } \\
\text { Hypoplasia/aplasia of metacarpals and } \\
\text { phalanges }\end{array}$ & $\begin{array}{l}+ \\
+ \\
+ \\
+\end{array}$ & $\begin{array}{l}+ \\
+ \\
+ \\
+\end{array}$ & $\begin{array}{l}+ \\
+ \\
+ \\
+\end{array}$ & $\begin{array}{l}+ \\
+ \\
+ \\
+\end{array}$ & $\begin{array}{l}+ \\
+ \\
+ \\
+\end{array}$ & $\begin{array}{l}? \\
+ \\
+ \\
+ \\
+\end{array}$ & $\begin{array}{l}? \\
+ \\
+ \\
+ \\
+\end{array}$ \\
\hline $\begin{array}{l}\text { Pelvis } \\
\text { Hypoplasia of pelvic bones }\end{array}$ & + & + & + & + & + & $?$ & $?$ \\
\hline $\begin{array}{l}\text { Lower limbs } \\
\text { Short, bowed/separated hypoplastic/aplastic } \\
\text { femur } \\
\text { Aplasia of fibula } \\
\text { Hypoplasia/aplasia of tarsals, metatarsals, and } \\
\text { phalanges }\end{array}$ & $\begin{array}{l}+ \\
+ \\
+\end{array}$ & $\begin{array}{l}+ \\
+ \\
+\end{array}$ & $\begin{array}{l}+ \\
+ \\
+\end{array}$ & $\begin{array}{l}+ \\
+ \\
+\end{array}$ & $\begin{array}{l}+ \\
+ \\
+\end{array}$ & $\begin{array}{l}+ \\
+ \\
+\end{array}$ & $\begin{array}{l}+ \\
+ \\
+\end{array}$ \\
\hline $\begin{array}{l}\text { Thorax } \\
\text { Pseudarthrosis of clavicle } \\
\text { Broad clavicles } \\
\text { Broad ribs }\end{array}$ & $\begin{array}{l}+ \\
- \\
-\end{array}$ & $\begin{array}{l}+ \\
- \\
-\end{array}$ & $\begin{array}{l}- \\
- \\
-\end{array}$ & $\begin{array}{l}- \\
- \\
-\end{array}$ & $\begin{array}{l}- \\
+ \\
+\end{array}$ & $\begin{array}{l}- \\
+ \\
+\end{array}$ & $\begin{array}{l}- \\
+ \\
+\end{array}$ \\
\hline
\end{tabular}

$+=$ present; $-=$ not present or not mentioned; ? = too early to know. 

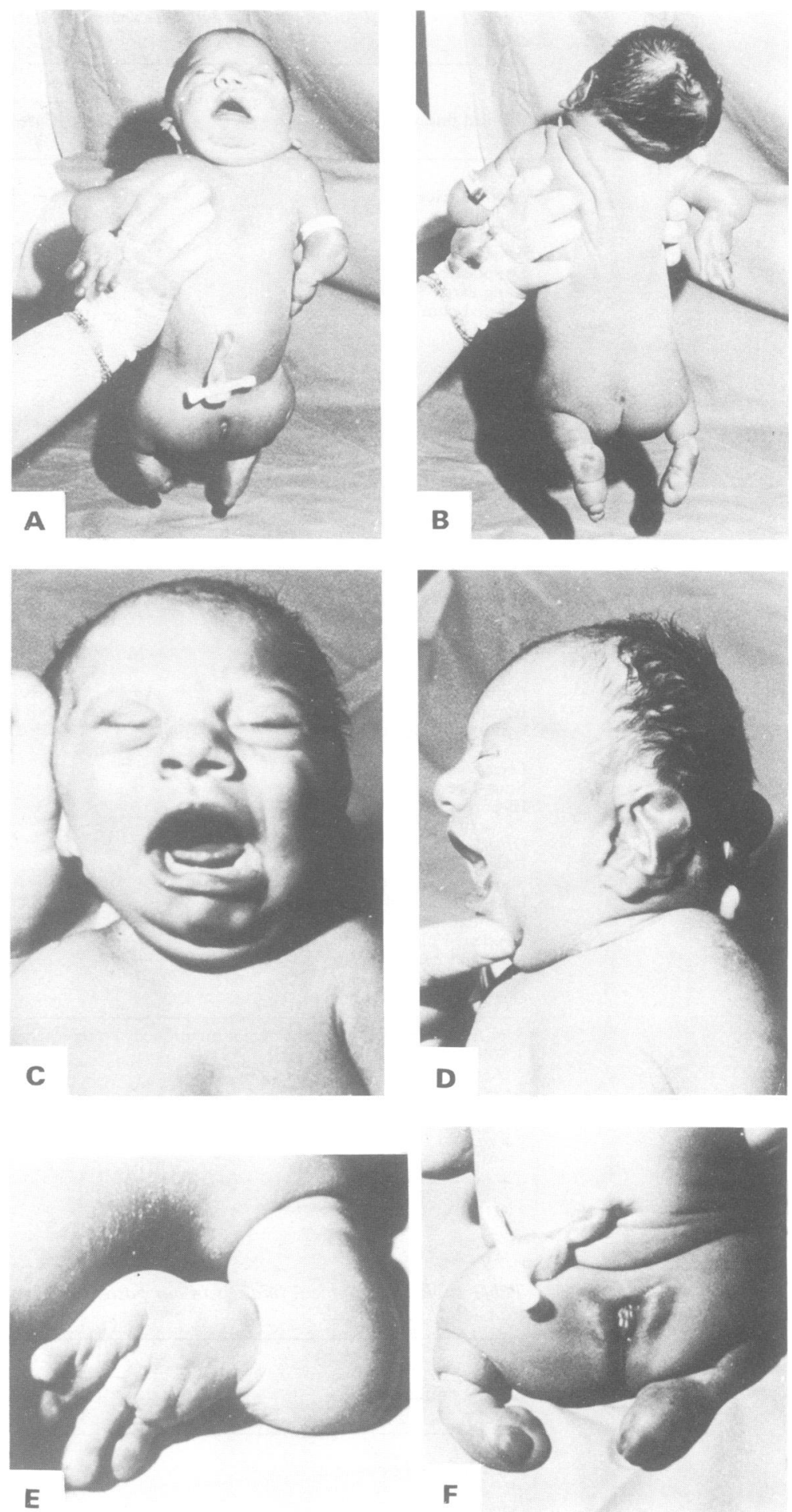

Figure 4 Case 2. ( $A, B, C)$ patient's appearance, (D) low set dysplastic ear, $(E)$ malformed upper limb and hand, $(F)$ anteverted external genitalia and stick-like appendages with malformed feet.

and narrow palate, and pseudarthrosis of the right clavicle. Ultrasonographic examination showed no defects of the internal organs. Agenesis of a kidney, meningocele, and hypoplasia of the cerebellum have been associated with limb/pelvis-hypoplasia/aplasia syndrome. This condition shows autosomal recessive inheritance. The patients described by Al-Awadi et $a l^{2}$ and those reported by Raas-Rothschild et $a l^{1}$ had consanguineous parents. The two sibs reported here are a further confirmation of autosomal recessive inheritance. It has been suggested that the gene responsible for this rare syndrome could be confined to the Middle East gene pool. ${ }^{1}$ However, these two Italian neonates affected with the limb/pelvis-hypoplasia/aplasia syndrome argue against this.

The parents of our patients and those of the 


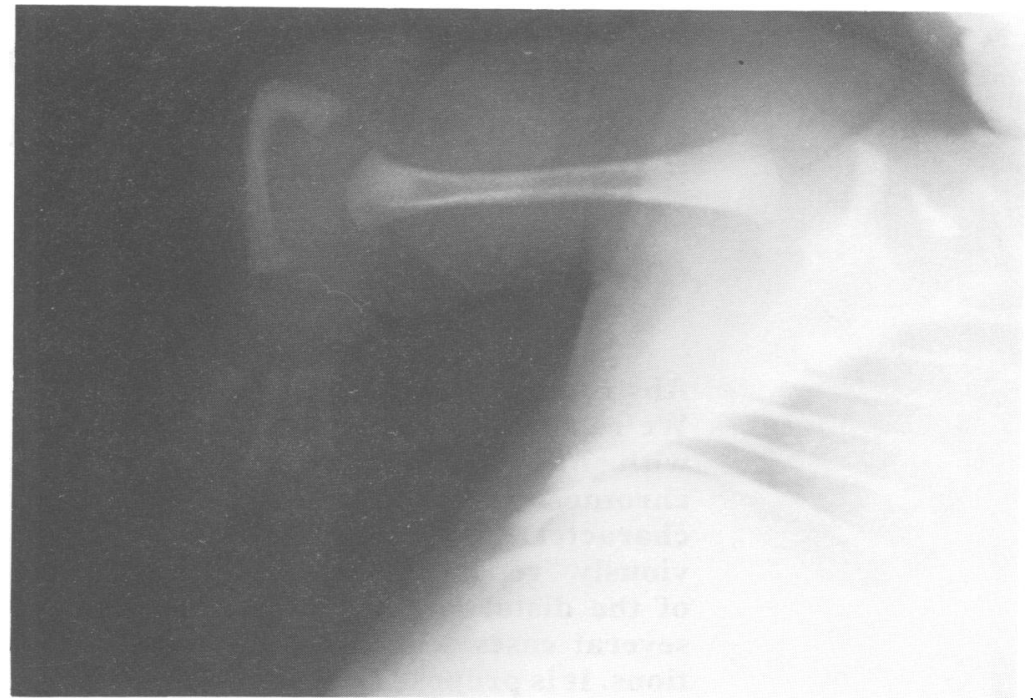

Figure 5 Case 2. Pseudarthrosis of clavicle, bowed radius, absent ulna, and four malformed digits.

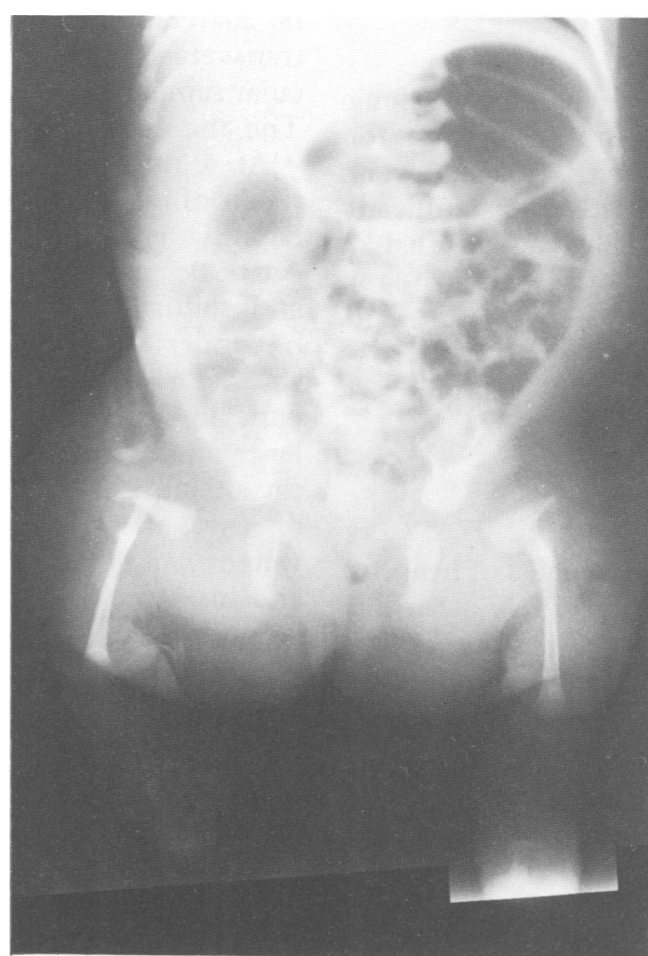

Figure 6 Case 2. Hypoplasia of pelvic bones, hypoplastic femora, short and slender tibiae, and absent fibulae. other patients are healthy and show no defects, so that the heterozygous state is impossible to detect.

We propose that this syndrome should be called the Al-Awadi/Raas-Rothschild syndrome.

We thank Dr M Baraitser, London, for his helpful criticism.

1 Raas-Rothschild A, Goodman RM, Meyer S, et al. Pathological features and prenatal diagnosis in the newly recognised limb/pelvis-hypoplasia/aplasia syndrome. $f$ Med Genet 1988;25:687-97.

2 Al-Awadi SA, Teebi AS, Farag T, Naguib KM, El-Khalifa MY. Profound limb deficiency, thoracic dystrophy, unusual facies, and normal intelligence: a new syndrome. $\mathfrak{f}$

3 Aitken GT. Proximal femoral focal deficiency: definition, classification, and management. In: Aitken GT, ed. Proximal femoral deficiency: a congenital anomaly. Washington, DC: National Academy of Sciences, 1969:1-22.

4 Kuhne D, Lenz W, Petersen D, Sehonenberg H. Defekt von femur und fibula mit amelie, peromelie oder ulnaren strahldefekten der arme. Ein syndrom. Humangenetik 1967;3:244-63.

5 Daentl DL, Smith DW, Scott CI, Hall BD, Gooding CA. Femoral hypoplasia-unusual facies syndrome. $\mathcal{f}$ Pediatr 1975;86:107-11. 